\title{
Structural Model for the Analysis of Stock Market Price Index
}

\author{
Agwuegbo, S.O.N. \\ Department of Statistics, University of Agriculture, Abeokuta, Nigeria \\ Mojekwu, J.N. (Corresponding author) \\ Department of Actuarial Science and Insurance, University of Lagos, Nigeria \\ E-mail: jnmoje@yahoo.com
}

Adewole, A.P.

Department of Computer Science, University of Lagos, Nigeria

Maduegbuna, A.N.

Department of Banking and Finance, Paul University Awka

Received: August 26, 2011 Accepted: September 7, $2011 \quad$ Published: November 1, 2011

doi:10.5539/ijms.v3n4p17 URL: http://dx.doi.org/10.5539/ijms.v3n4p17

\begin{abstract}
Stock market returns are predictable from a variety of financial and macroeconomic variables and have long been an attraction for equity investors. Recently increasing attention has shifted on the market index as a method of measuring a section of the stock market. The stock market index is regarded as an important indicator by the investing public at large and can be used as a benchmark by which investor or fund manager compares the returns of their own portfolio.

In this study, attempts are made to model the Nigerian stock market index using a structural model. The procedure is based on the relationship between the state space and the autoregressive moving average (ARMA) model. The time series procedure from S-PLUS software is used in the analysis. The result obtained shows that the Nigerian stock market price index is an autoregressive model (AR) of order 1. It is also found that the AIC is at minimum at lag 1 which corresponds to the same model identified for the series by using the sample ACF and PACF.
\end{abstract}

Keywords: Dynamical system, Filter, Markov process, Signal process

\section{Introduction}

Financial time series such as stock market returns exhibit certain patterns which are crucial for correct model specification, estimation and forecasting. Stock market returns are predictable from a variety of financial and macroeconomic variables and has long been an attraction for equity investors. Recently increasing attention has shifted on the market index as a method of measuring a section of the stock market. The stock market index are regarded as an important indicator by the investing public at large and can be used as a benchmark by which investor or fund manager compares the returns of their own portfolio.

The stock market index is a way of measuring the performance of a market over time. Forecasting the stock market index is an important financial problem that is receiving increasing attention, since the market index represents the return of the market as a whole. The market index calls for serious examination due to the fact that it defines the universe from which investors or fund managers pick their stock. There are lots of irregularities in defining the stock market index, making it difficult to define the path of the process. These sudden structural changes can be reduced by the use of a structural model. A structural model, Harvey (1989) sets out to capture the salient feature of a time series. Discussion of the methodological and technical ideas underlying the structural time series models can be found in Harvey (1989) and Chatfield (2004). 
Structural models can be written as a state space model with the state of the system, representing the various unobserved components and the parameters (structural parameters) having clear interpretations. The state space representation of a system is a fundamental concept in modern control theory (Wei, 1990). The state of a system is defined to be a minimum set of information from the present and past such that the future behaviour of the system can be completely described by the knowledge of the present state and the future input. The State space representation is based on the Markov property, which implies that given the present state, the future of a system is independent of its past. A Markov property is built on the presumption that the future will in some sense be like the past. There is flexibility of these models where one can specify a probability law consistent with a broad range of different outcomes, and choose particular parameters within that class on the basis of data.

One advantage obtained by modeling the changes in terms of strictly stationary Markov chains as opposed to using a deterministic function is that the observations generated by a stochastic process may still under certain conditions be a strictly stationary and ergodic process, even though a particular realization of the process may have a non-stationary outlook. The discrete Markov assumption implies that the parameter states occur repeatedly over the available data which makes the estimation more efficient since several data sections can be combined to yield the estimate of a given parameter value.

In this article, we introduce a class of structural models in order to capture the salient feature of the stock market index in Nigeria. The structural model is reduced as an autoregressive moving average (ARMA) process. Akaike (1974) was the first to demonstrate that structural models can be reduced to ARMA (p,q) model. The relationship between the structural model and its reduced forms gives considerable insight into the potential effectiveness of the different ARMA models (Harvey, 1989; Chatfield, 2004). ARMA models, typically are parsimonious model (Box and Jenkins, 1976, Box et al, 1994) and is based on the premise that the autocorrelation function (ACF) and the related statistics can be accurately estimated and are stable over time. By adopting Box-Jenkins ARMA approach to time series analysis, model identification, parameter estimation and diagnostic checks are feasible for the analysis of Nigerian stock market price Index.

\section{Materials and Methods}

The Nigerian stock market price Index was examined by using a basic structural time series modeling approach. The key to handling structural time series models is the state space form with the state of the system representing the various unobserved components such as trends and seasonal. The prime objective of state space model is to estimate the signal in the presence of noise. The state space approach to time series model focused attention on the state vector of a system. The measurement vector represents noisy observations that are related to the state vector. It is assumed that the noise contaminates the signal in an additive manner so that the actual observations are given by the following measurement equation

$$
Z_{t}=F_{t} \theta_{t}+V_{t} \quad V_{t} \sim N\left(0, \sigma_{e}^{2}\right)
$$

where $Z_{t}(t=1,2, \ldots \ldots \ldots \ldots . . . N)$ is the observed noise corrupted time series, $F_{t}$ is assumed to be an $((n \times 1)$ known column vector, $\theta_{t}$ and $V_{t}$ are the time series representing an $(n \times 1)$ state vector and the observation noise respectively. The vector $\theta_{t}$ may not be directly observable. It is often assumed as a vector difference equation or state equation represented as

$$
\theta_{t}=H_{t} \theta_{t-1}+W_{t}
$$

where the $(n \times n)$ matrix $H_{t}$ is assumed known, and $W_{t}$ denotes an $(n \times 1)$ vector of deviations such that $W_{t}^{T}=\left(w_{1, t}, w_{2, t}, \ldots \ldots \ldots . . . w_{n, t}\right)$.

The pair of equations in (1) and (2) constitute the general form of the state space model. The errors in the measurement (or observation) equation in (1) and state (or transition) equation in (2) are generally assumed to be serially uncorrelated and also to be uncorrelated with each other at all time periods. Further, the measurement error $V_{t}$ is assumed as an independent random Gaussian process while $W_{t}$ is a white Gaussian noise with zero mean and variance matrix $\sigma_{w}^{2}$. Additionally $V_{t}$ and $W_{t}$ are assumed to be orthogonal at all pairs of time.

\section{Estimation of the Structural Parameters}

The estimation of the parameters and the state vectors efficiently can be calculated by the Kalman filter, which is an important general method of handling state-space models. Essentially Kalman filtering is a method of signal processing, which provides optimal estimates of the current state of a dynamic system (Chatfield, 2004). Kalman (1960) defined filtering as any mathematical operation which uses past data or measurements on a given dynamical system to make more accurate statement about present, future or past variables in that system. Kalman filter degenerates into simpler algorithm that is identical with the conventional time series method of 
forecasting. The importance of the Kalman algorithm is based on the fact that it constitutes the main procedure of estimating dynamic systems represented in state space form. For the linear Gaussian estimation problem, the required probability density function (pdf) remains Gaussian at every iteration of the filter, and the Kalman filter, propagate and update the mean and covariance of the distribution (Chatfield, 2004).

The Kalman filter recursively evaluates the estimator of the state vector conditional on the past observations up to time $(t-1)$. By considering Equation (2), where $W_{t}$ is still unknown at time $t-1$, the obvious estimator for $\theta_{t}$ is given as

with variance covariance matrix

$$
\hat{\theta}_{t / t-1}=H_{t} \hat{\theta}_{t-1}
$$

$$
P_{t / t-1}=H_{t} P_{t-1} H_{t}^{T}+W_{t}
$$

Equations (3) and (4) are the prediction equations. Equation (4) follows from standard results on variance -covariance matrices for vector random variables (Chatfield, 2004; Stark and Woods, 1986). When new observation has been observed, the estimator for $\theta_{t}$ can be modified to take account of this extra information. At time $(t-1)$, the best forecast of $Z_{t}$ is given as $F_{t} \hat{\theta}_{t / t-1}$ so that the prediction error is given by

$$
\varepsilon_{t}=Z_{t}-F_{t} \hat{\theta}_{t / t-1}
$$

$\varepsilon_{t}$ in (5) is called the prediction error. This quantity can be used to update the estimate of $\theta_{t}$ and of its variance-covariance matrix and the best way to do this is by means of the following equation

$$
\hat{\theta}_{t}=\hat{\theta}_{t / t-1}+K_{t} \varepsilon_{t}
$$

and

where

$$
P_{t}=P_{t / t-1}-K_{t} F_{t} P_{t / t-1}
$$

$$
K_{t}=P_{t / t-1} F_{t}\left[F_{t} P_{t / t-1} F_{t}^{T}+\sigma_{v}^{2}\right]^{-1}
$$

$K_{t}$ in (8) is called the Kalman gain matrix and is a vector of size $(m \times 1)$. Equation (6) and (7) constitute the second updating stage of the Kalman filter and are called the updating equations.

\section{Results}

The plot of the Nigerian stock market price index is as in Figure 1. The time plot in Figure 1 revealed that the Nigerian stock market price index is nonstationary. The time plot was achieved through the use of Splus software.

The first step in state-space modeling is to find the optimal autoregressive (AR) model that fits the data. The selection of a tentative time series model is frequently accomplished by matching estimated autocorrelations with the theoretical autocorrelation and partial autocorrelation functions. Table 1 is the ACf and the PACF of the Nigerian Stock market price index.

Based on the ACF and PACF of the market price index in Table 1, one may suggest an AR (1). The Splus package use the Akaike's Information Criterion (AIC) to provide an optimal or best fit for the autoregressive model. The value of the AIC is as in Table 2, and is minimum at $p=1$.

Hence the optimal AR order $p$ is chosen to be one. The Gauss Markov signal model generated from the stock market price index data using ARMA model is

$$
\theta_{t}=0.977 \theta_{t-1}+W_{t} \quad t \geq 0
$$

With mean equal to zero and $\sigma_{w}^{2}=0.00077$. The Kalman gain $K_{t}$ as defined in (8) is $K_{t}=0.027$, while the prediction error variance as defined in (5) is $\varepsilon_{t}=0.028$. The Kalman filter is asymptotically given by

$$
\hat{\theta}_{t \mid t}=0.951 \theta_{t-1 \mid t-1}+0.027 Z_{t} \text {. }
$$

\section{Discussion}

A structural model approach was developed for the analyses of the Nigerian stock market index. The procedure is based on the state space modeling technique. The state space formulation yields a practical means of estimation for this complex time varying dynamical process. Theoretically, there is no distinction between the state space model and the ARMA representations of a stationary process. An advantage of the state space models is that observations can be added one at a time, and the estimating equations are then updated to produce new estimates. The state space representation of a system is related to Kalman filter. The Kalman filter technique is 
adopted in this study as the best linear filter in an expected square error sense, and the filter algorithm degenerates into simpler algorithm that is identical with the conventional time series method of forecasting.

\section{References}

Akaike, H. (1974). A new look at the statistical model identification. IEEE Trans. Automat. Control, 19, 716-723. http://dx.doi.org/10.1109/TAC.1974.1100705

Box, G.E.P., and Jenkins, G.M. (1976). Time series analysis, forecasting and control (rev.ed). San Franscisco, Holden-Day.

Box, G.E.P., Jenkins, G.M., and Reinsel, G.C. (1994). Time series analysis, forecasting and control. (3rd ed.). Englewood Cliffs, N.J, Prentice-Hall.

Chatfield, C. (2004). The analysis of time series, an introduction. (6th ed.). Chapman and Hall/CRC, Boca Raton.

Harvey, A.C. (1989). Forecasting, structural time series models and the Kalman filter. Cambridge Univ. Press, Cambridge.

Kalman, R.E. (1960). A new approach to linear filtering and prediction problems. Journal of Basic Eng, Vol.82, pp. 35-45.

Stark, H., and Woods, J.W. (1986). Probability, Random Process, and Estimation Theory for Engineers. Prentice-Hall, Englewood Cliffs, New Jersey.

Wei, W.W.S. (1990). Time Series Analysis: Univariate and Multivariate Methods. Addison-Wesley Redwood City, CA.

Table 1. ACF and PACF of the Nigerian Stock Market Price Index

\begin{tabular}{|l|l|l|l|l|l|l|l|l|l|l|l|}
\hline Lag & 1 & 2 & 3 & 4 & 5 & 6 & 7 & 8 & 9 & 10 & 11 \\
\hline ACF & 0.894 & 0.795 & 0.698 & 0.600 & 0.499 & 0.395 & 0.301 & 0.240 & 0.187 & 0.153 & 0.117 \\
\hline PACF & 0.894 & -0.02 & -0.04 & -0.07 & -0.07 & -0.09 & -0.03 & 0.104 & -0.01 & 0.06 & -0.05 \\
\hline
\end{tabular}

Table 2. AIC of the Nigerian Stock Market Price Index

\begin{tabular}{|l|l|l|l|l|l|l|l|l|l|l|l|}
\hline Lag & 0 & 1 & 2 & 3 & 4 & 5 & 6 & 7 & 8 & 9 & 10 \\
\hline AIC & 94.47 & 0.00 & 1.97 & 3.86 & 5.60 & 7.31 & 8.83 & 10.79 & 12.15 & 14.14 & 15.96 \\
\hline
\end{tabular}




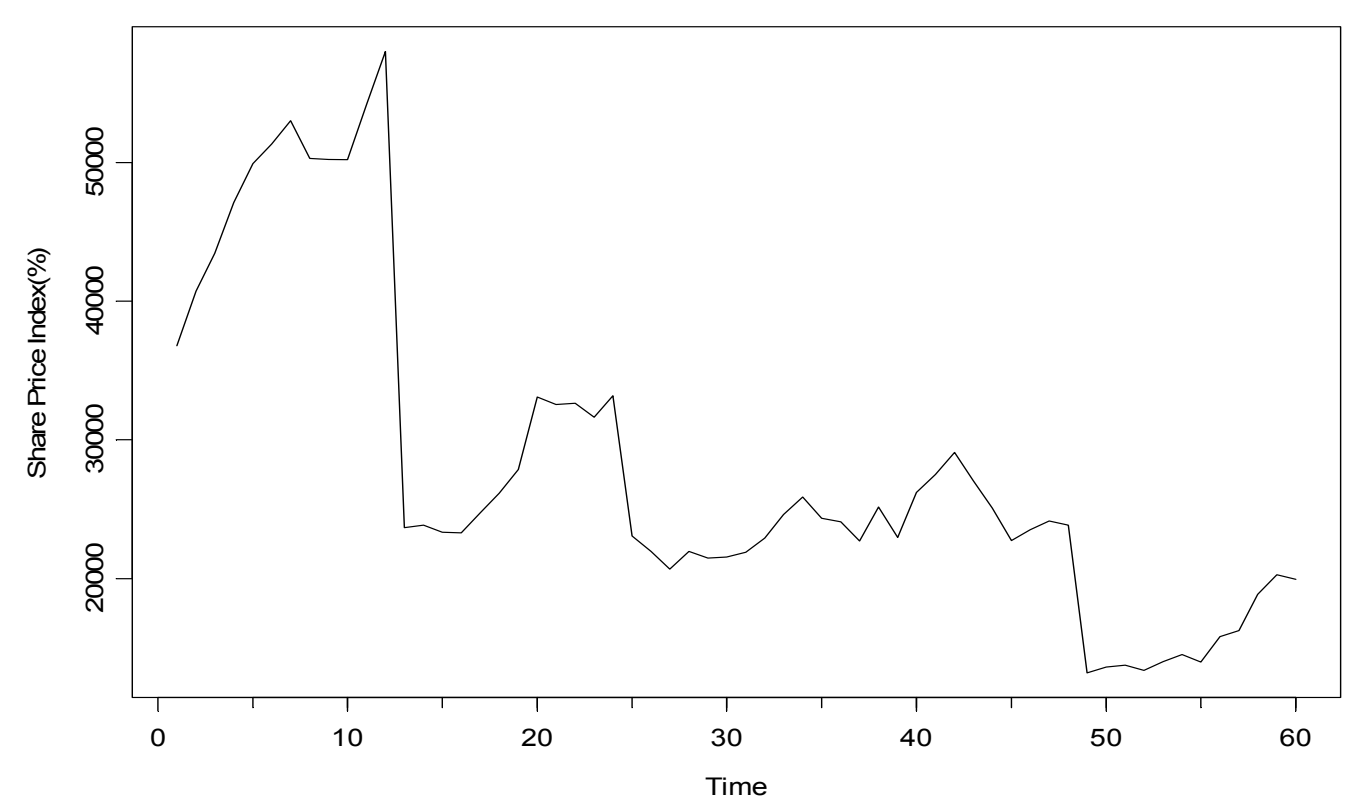

Figure 1. Time Plot of the Nigerian Stock Market Price Index

\section{Series : Share Price Index.ts}

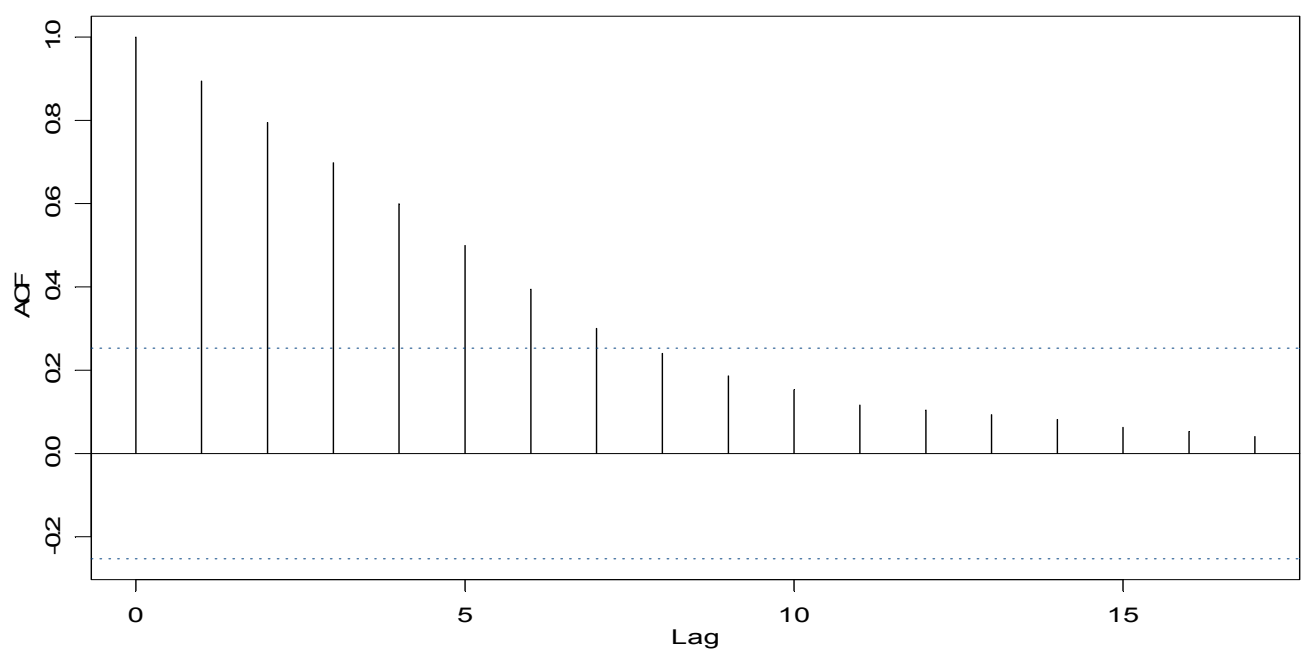

Figure 2. ACF of the Nigerian Stock Market Price Index 


\section{Series : Share Price Index.ts}

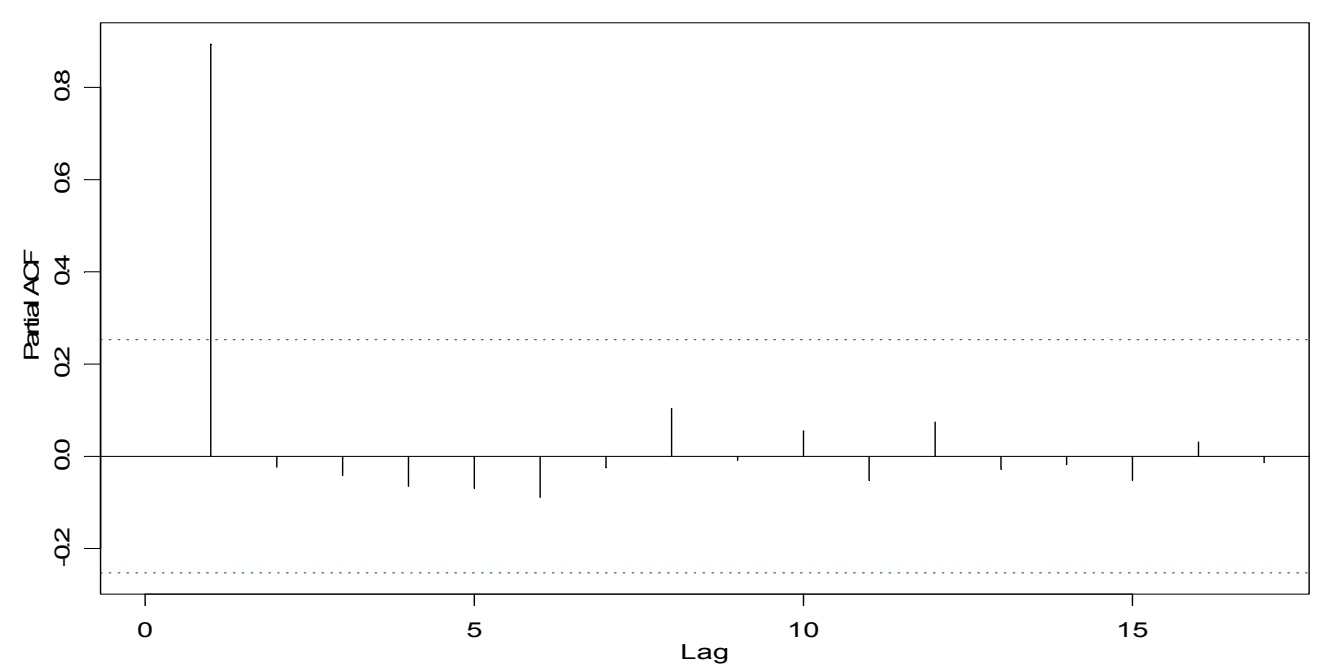

Figure 3. PACF of the Nigerian Stock Market Price Index 